\title{
How to avoid drug resistance during treatment and prevention of urinary tract infections
}

\author{
Damian Warzecha, Bronisława Pietrzak, Aleksandra Urban, Mirosław Wielgoś
}

$1^{\text {st }}$ Department of Obstetrics and Gynaecology, Medical University of Warsaw, Warsaw, Poland

\begin{abstract}
Urinary tract infections (UTIS), defined as the presence of bacteria above the bladder sphincter, are among the most common infectious diseases. They remain a significant cause of antibiotic prescription worldwide. The incidence is much higher among women, especially of reproductive age, than among men. If the infection occurs at least 3 times a year or twice within 6 months, it is classified as recurrent urinary tract infection (rUTI). Among the causal pathogens, the vast majority are Gram-negative bacteria, the most common of which is Escherichia coli. Recommended treatment regimens differ depending on the diagnosed disease entity and the patient's clinical situation. Empirical antibiotic therapy is most often used. The first-line treatment in patients with acute simple cystitis include nitrofurantoin, trimethoprim-sulfamethoxazole, or fosfomycin. Beta-lactams and fluoroquinolones should be considered as a second-line agent. In particular cases (pregnancy or rUTIs) targeted treatment, based on the results of urine culture and antibiogram, is implemented. During pregnancy recommended treatment includes administration of cephalosporins (e.g. cefuroxime) or nitrofurantoin. In patients with uncomplicated pyelonephritis fluoroquinolones should be considered as the first-line regimen.

In the case of rUTIs, there are no uniform guidelines for prophylactic management. Repeated administration of antibiotics due to infections leads to a growing problem of drug resistance. Most recommendations suggest not to use antibiotic prophylaxis routinely. Growing evidence favours non-antibiotic prophylaxis regimens for recurrent UTIs. Until now only one product - oral immunostimulant OM-89 - has been sufficiently investigated. Wider implementation of immunoprophylaxis in the future may reduce possible side effects of inappropriate antibiotic consumption.
\end{abstract}

Key words: urinary tract infections, pregnancy, antibiotics, immunoprophylaxis.

\section{Introduction}

Urinary tract infections (UTIS) remain the leading cause of complaints in outpatient clinics and the most common infectious diseases among women of reproductive age [1]. This term defines various disease entities as cystitis or pyelonephritis. Every year, approximately $15 \%$ of sexually active women have an episode of UTIs, and up to $60 \%$ of them develop UTIs at some time in their life. The typical symptoms of simple infection include: suprapubic pain, polyuria, urinary frequency and urgency, painful micturition, and, in case of spreading the infection into the upper urinary tract: fever, flank pain, chills, and malaise. On the other hand, asymptomatic bacteriuria $(A B)$ refers to isolation of bacteria in the urine of patient without any symptoms of UTIs. The most common pathogen isolating from the patients with the signs of UTIS or $A B$ is the endogenous intestinal flora, especially Escherichia coli [2]. When it occurs at least twice every 6 months or 3 times a year and follows the resolution of a previous UTI it is classified as a recurrent urinary tract infection (rUTIs). Most often the infection is uncomplicated - it affects non-pregnant women, without anatomical or functional urinary tract defects or comorbidities. It is clinically significant to distinguish complicated UTIs, which are associated with an increased risk of severe course. It occurs, among others, in pregnant women, all men, patients with anatomical or functional abnormalities of the urinary tract, impaired antimicrobial defence mechanisms, renal diseases, inserted indwelling medical devices, or when UTIs are caused by atypical microorganisms

The diagnosis of UTIs is usually made on the basis of reported signs and symptoms supported by laboratory tests (urinalysis and urine culture). The presence of $\geq 10^{5}$ bacteria per millilitre (colony forming units per millilitre - CFU/ml) confirms the infection, while the antibiogram allows us to verify the effectiveness of the therapy. Urine culture prior to therapy implementation is not obligatory in patients without any known risk fac- 
tors of complicated UTIs (urologic abnormalities, immunosuppression, or poorly controlled diabetes mellitus); however, it reduces the possible development of drug resistance among uropathogens. It is highly advised when acute pyelonephritis or pregnancy is suspected or if the symptoms do not resolve or recur within 4 weeks after the completion of previous treatment. On the other hand, most infections occur in sexually active women without any identifiable predisposing condition. Close proximity of the reproductive tract indicates that the inflammatory process in the vagina is also well-known risk factor of urinary infections. It is estimated that more than half of UTIs in females are accompanied or preceded by vaginitis [3].

The following paper is an attempt to summarize the current state of the art regarding the treatment and prevention of UTIs in women to avoid the possibility of developing drug resistance by causal strains in the future.

\section{Description of the current state of knowledge}

\section{Treatment of urinary tract infections}

The choice of first-line therapy differs, depending on the diagnosed disease entity and the patient's clinical situation.

The first-line treatment in patients with acute simple cystitis depends on the possible risk of infection with a multidrug-resistance (MDR) pathogen. Antibiotics targeted toward Escherichia coli, Proteus, Klebsiella, and Staphylococcus saprophyticus are the recommended first-line treatment. Empirical treatments are often eligible and include nitrofurantoin, trimethoprim-sulfamethoxazole, or fosfomycin [4, 5]. Beta-lactams and fluoroquinolones should be considered as second-line agents due to possible induction of MDR and higher risk of side effects than the aforementioned antimicrobials [6]. In patients with contradictions to the first-line agents or high risk of MDR Gram-negative infection, directed antimicrobial therapy should be introduced. Delayed antibiotic therapy based on the urine culture result is also acceptable.

The first-line treatment in patients with uncomplicated pyelonephritis who do not require hospitalization, should be commenced with a short course of fluoroquinolones.

Asymptomatic bacteriuria should be screened and treated if detected only in pregnant women and in patients prepared to urological procedures breaching the mucosa [7]. Targeted therapy should be preceded by a urine culture.

Pregnancy, especially if complicated by gestational diabetes mellitus, is a well-known risk factor for urinary infection. An increase in progesterone concentration leads to the relaxation of smooth muscles and ureteral dilatation. In combination with immune changes causing the state of relative immunocompromise, it predisposes pregnant women to UTIs [8]. Treatment of any kind of UTI as well as AB in gravid patients should always be targeted due to the high risk of further complications. It means that urine culture with an antibiogram must be performed prior to the introduction of antibiotics. It is estimated that pyelonephritis occurs in up to $2 \%$ of pregnancies. In this group of patients, early introduction of broad-spectrum antibiotic therapy is required. The route of administration and the possible management (outpatient vs. inpatient) depend on the baseline condition of the patient.

First-line therapy during pregnancy should include administration of cephalosporins (e.g. cefuroxime) or nitrofurantoin (avoided at term due to possible neonatal haemolysis) [9]. Amoxicillin with clavulanic acid should be carefully used in the third trimester due to the risk of necrotizing enterocolitis in newborns.

The duration of treatment varies by specific drug and type of infection, ranging from 3-7 days in uncomplicated cystitis to 7-14 days in pyelonephritis [10]. However, in the case of uncomplicated UTI treatment, 3 days of antibiotic therapy seem to have similar effectiveness as 5-10 days in achieving resolution of the symptoms [11].

\section{Treatment and prophylaxis of recurrent urinary tract infection}

The implementation of optimal therapy is extremely important in the case of recurrent UTIs. Of the $60 \%$ of women who develop a UTIs in their lifetime, $30 \%$ will struggle with rUTIs. Moreover, it is estimated that up to $20 \%$ of pregnant women will experience UTIs, with the recurrence rate of UTIs up to $23 \%$ during the same pregnancy or soon after the birth [8].

Recurrent urinary tract infection may cause serious adverse pregnancy outcomes for both mother and child. Meta-analyses have demonstrated that the occurrence of infection during pregnancy increases the risk of preeclampsia [12]. Moreover, the research also provided evidence that the consequence of the infection is lipopolysaccharide-induced damage to the red blood cell membranes clinically manifested by anaemia [13]. Other conditions proven to complicate acute pyelonephritis in pregnant women include preterm labour, intrauterine growth restriction, and premature rupture of membranes. The last 2 mentioned occur more often, despite rapid and adequate therapy implementation [14]. Routine urinalysis screening and early treatment of detected UTIs should be an integral part of pregnancy management because they enable the prevention of certain complications. 
The aetiological factor of the recurrent UTIs should always be diagnosed by urine culture [7].

As well from the aforementioned factors, women diagnosed with rUTIs more often suffer from chronic anxiety, fear, and depressed mood resulting from the unexpected, sudden, and distressing character of UTI episodes [15]. On the other hand, effective prophylaxis reducing recurrence rates improves individuals' quality of life. Therefore, effective prevention without severe side effects that may impact the individual's wellbeing would be the best choice.

Insufficient evidence supports the best way to prevent rUTIs. The available interventions that may impact the recurrence rates include pharmacological (antibiotics, immunostimulants) or non-pharmacological (cranberry products, probiotics, behavioural modifications) prophylaxis. All patients should be counselled on the possible risk factors for recurrent infections [16].

Nowadays, due to the possibility of inducing drug resistance among pathogenic strains and the greater number of contraindications, continuous or postcoital antimicrobial treatment as a prophylaxis against recurrent UTIs should be introduced only when available nonantimicrobial interventions have failed.

\section{Pharmacological prophylaxis}

\section{Immunoprophylaxis}

Vaccines and immunostimulants seem to be the most promising options in the near future. The idea is to stimulate the host's immune system with the most common pathogens to enhance defence mechanisms against UTIs. Mucosa-associated lymphoid tissue plays a crucial role in humoral and cellular immune response in the urinary tract. Activation of dendritic cells as well as continuous presentation of the antigens seem to be the most rational targets for immunotherapy, based on its unique ability to induce immunity.

Until now only one product - oral immunostimulant OM-89 (Uro-Vaxom) - has been sufficiently investigated to draw clear recommendations in accordance with current standards. Lyophilized lysate of 18 Escherichia coli strains enhances both innate and acquired immunity (humoral and cellular one) against the most common aetiological factors of urinary infections. Beerepoot et al. assessed the effectiveness, tolerability, and safety of nonantibiotic prophylaxis in adults with rUTIs. According to the available data, the authors concluded that OM89 decreased the rate of recurrence (RR $0.61,95 \% \mathrm{Cl}$ : 0.48-0.78) with a good safety profile [17]. After 6 months the mean difference in the recurrence rates between the study group and placebo equalled -0.26 (95\% Cl: -0.36 to $-0.16, p=0.0004)$ [18]. Significant reduction in the number of UTIs within the year following initiation of immunostimulant compared to the year preceding administration (3.14 vs. 1.53, $p<0.05$ ) was observed by Brodie et al. [19]. Oral immunization with OM-89 is recommended by the European Society of Urology (EAU) (Fig. 1) and by the Polish Society of Urology in cases of uncomplicated rUTIs (strong evidence, highest level of recommendation - $1 \mathrm{~A}$ ), in order to reduce the recurrence rates, severity of reported symptoms, the amount of prescribed antibiotics, and the possible MDR strains development in the future [7]. The drug is available orally, due to its resistance to intestinal proteases. It is administered once a day, on an empty stomach (30 minutes before a meal), for 90 days in order to prevent recurrent UTIs. Moreover OM-89 is generally safe and well tolerated by patients. No serious side effects were reported so far. It can be safely administered to immunocompromised patients, elderly people, and women in the second and third trimesters of pregnancy.

\section{Oestrogens in postmenopausal women}

Hypoestrogenism, resulting from menopausal transition, is another well-known risk factor related with UTIs. Recurrent infections occur in every fifth women after menopause. Exogenous oestrogens contribute to the restitution of the normal vaginal ecosystem, which poses the theoretical justification for their use in the prevention of rUTIs in this group of patients. Long-term vaginal oestrogen replacement therapy in older women effectively reduces the amount of symptomatic UTI episodes by more than $50 \%[20,21]$.

All the aforementioned contents indicate the necessity for further investigations in order to select the most effective and possibly least invasive preventive strategy of rUTI management. Non-pharmacological treatment should be recommended for all women with recurrent UTIs.

\section{SEQUENCE OF THERAPEUTIC MANAGEMENT IN RECCURENT URINARY TRACT INFECTIONS ACCORDING TO EUROPEAN SOCIETY} OF UROLOGY 2020 GUIDELINES

\begin{tabular}{l|c|c}
\multicolumn{1}{c|}{ STEP I } & STEP II & STEP III \\
\hline $\begin{array}{l}\text { Diagnose recurrent UTI by urine culture } \\
\text { Introduce behavioural modifications }\end{array}$ & $\begin{array}{c}\text { Non-antimicrobial prophylaxis: } \\
\text { immunoactive prophylaxis with OM-89 - level } \\
\text { of evidence 1 A, recommendations: strong } \\
\text { vaginal estrogen replacement in postmenopausal } \\
\text { women - level of evidence 1 B, recommendation: } \\
\text { weak }\end{array}$ & $\begin{array}{l}\text { Antimicrobial prophylaxis } \\
\text { (only if STEP I and II are ineffective): } \\
\text { pontinuous low-dose antimicrobial }\end{array}$ \\
posital prophylaxis
\end{tabular}

Fig. 1. Therapeutic management in recurrent urinary tract infections according to European Society of Urology 2020 guidelines UTI - urinary tract infections 


\section{Antibiotic prophylaxis}

Continuous antibiotic prophylaxis may be considered in patients with recurrent cystitis not related to sexual activity. Regular long-term prevention with the usage of nitrofurantoin, trimethoprim-sulfamethoxazole, or cephalexin medications is an option in nonpregnant women. The dose of antibiotics is generally lower than that given in acute infection (nitrofurantoin 50-100 mg once daily, trimethoprim-sulfamethoxazole 240-480 mg once daily, or cephalexin 125-250 mg once daily). A comprehensive metanalysis prepared by Albert et al. proved that prophylactic antibiotics used to prevent uncomplicated rUTIs in adult non-pregnant women significantly (more than 6-fold) reduced the recurrence rates (RR was $0.15,95 \% \mathrm{Cl}: 0.08-0.28$ ) while the number needed to treat equalled 1.85 [22] On the other hand, another study showed that approximately $50 \%$ of patients develop recurrent cystitis with a similar frequency as before the antibiotic prophylaxis [23].

The recommendations of the World Health Organization regarding antenatal care emphasize that antibiotic prophylaxis in pregnant women may prevent recurrence relapse of UTIs [24]. However, according to the Cochrane Database, a daily dose of nitrofurantoin together with close surveillance has not been shown to prevent rUTIs compared with close surveillance alone [25]. The authors did not find any significant differences regarding recurrent pyelonephritis, rUTIs before birth, and preterm birth. Moreover, it is worth emphasizing that antibiotic prophylaxis may have an impact on the pregnancy-related outcomes and might increase the risk of antimicrobial resistance in the future. According to previous studies, the percentages of strains resistant to selected antibiotics are as follows: trimethoprim-sulfamethoxazole $-30.3 \%$, cefixime $9.9 \%$, pivampicillin $7.5 \%$, and nitrofurantoin $2.5 \%$ [9]. Systemic antibiotics may also lead to alternations in human microbiome, especially intestinal and genital tract. Although most therapeutics are well tolerated, the patients should be aware of potential drug toxicities. The most commonly reported complaints include gastrointestinal symptoms (nausea, vomiting, diarrhoea, or stomach pain), skin (rash, pruritus), as well as recurrent vaginal and oral candidiasis [22]. Trimethoprim sulfamethoxazole should be avoided during the first and third trimester of pregnancy, while nitrofurantoin cannot be used by patients with severe renal failure $[26,27]$.

\section{Nonpharmacological prophylaxis}

However, adequate phase III studies are necessary, effectiveness of behavioural changes as liberal fluid intake were proved in previous studies. Increased water uptake of an additional 1.5 litres a day reduces the recurrence of acute cystitis and decreases the risk of anti- microbial therapy use [28]. Other approaches such as postcoital micturition, hygienic habits, urinary alkalisers, or cranberry products, although routinely recommended, have not been proven to be beneficial [29]. In-vitro studies support the biological plausibility of cranberry juice, due to reduced adherence of uropathogens to uroepithelial cells and strain formulation [30,31]. Although some small studies have demonstrated encouraging results, the recent metanalysis suggests that it is less effective than previously indicated and should not be recommended for the prevention of UTIs [32].

Most previous studies regarding oral probiotics did not demonstrate significant reduction in the incidence of rUTIS [33]. On the other hand, the route of administration may be crucial. Vaginal administration of Lactobacillus decreased recurrence rates [34]. D-mannose has been used in the prevention of rUTIs. This preparation may prevent bacteria from adhering to the epithelium of the urinary tract; however, further studies are required.

\section{Conclusions}

In recent years empirical or targeted anti-infective agents together with long-term low-dose prophylactic antibiotics have been the cornerstone in the treatment and prevention of UTIs. However, against the growing drug resistance within the uropathogens, alternative non-antibiotic strategies are becoming an extremely attractive and reasonable solution. Antibiotics should be used for as short as possible, while the dosage and duration of therapy depend on the type of infection. Most recommendations, such as from the EAU, suggest not using antibiotic prophylaxis routinely as a first-line option. In the case of frequent relapses, urine culture prior to the therapy is highly advised. Growing evidence favours non-antibiotic prophylaxis regimens for recurrent UTIs. Wider implementation of immunoprophylaxis in the future may reduce possible side effects of inappropriate antibiotic consumption and may help in reducing drug resistance in causal strains.

\section{References}

1. Foxman B. Epidemiology of urinary tract infections: incidence, morbidity, and economic costs. Dis Mon 2003; 49: 53-70.

2. Hames L, Rice CE. Antimicrobial resistance of urinary tract isolates in acute uncomplicated cystitis among college-aged women: choosing a first-line therapy. J Am Coll Health 2007; 56: 153-156.

3. Rzepka-Gorska I, Sowinska E, Wilczynska E. [Urinary tract infections in relation to inflammatory conditions of the vagina in girls]. Ginekol Pol 1989; 60: 27-32.

4. Stein GE. Comparison of single-dose fosfomycin and a 7-day course of nitrofurantoin in female patients with uncomplicated urinary tract infection. Clin Ther 1999; 21: 1864-1872.

5. Little P, Moore MV, Turner S, et al. Effectiveness of five different approaches in management of urinary tract infection: randomised controlled trial. BMJ 2010; 340: c199. 
6. Hooton TM, Scholes D, Gupta K, et al. Amoxicillin-clavulanate vs ciprofloxacin for the treatment of uncomplicated cystitis in women: a randomized trial. JAMA 2005; 293: 949-955.

7. Bonkat G, Pickard R, Bartoletti R, et al. EAU guidelines on urological infections. European Association of Urology 2017: 22-26.

8. Habak PJ, Griggs Jr RP. Urinary tract infection in pregnancy. StatPearls 2019.

9. Czajkowski K, Bros-Konopielko M, Teliga-Czajkowska J. Urinary tract infection in women. Menopause Rev 2021; 20: 40-47.

10. Kolman KB. Cystitis and pyelonephritis: diagnosis, treatment, and prevention. Prim Care 2019; 46: 191-202.

11. Milo G, Katchman EA, Paul M, et al. Duration of antibacterial treatment for uncomplicated urinary tract infection in women. Cochrane Database Syst Rev 2005: CD004682.

12. Yan $\mathrm{L}$, Jin $\mathrm{Y}$, Hang $\mathrm{H}$, et al. The association between urinary tract infection during pregnancy and preeclampsia: A meta-analysis. Medicine (Baltimore) 2018;97(36):e12192.

13. Cox SM, Shelburne P, Mason R, et al. Mechanisms of hemolysis and anemia associated with acute antepartum pyelonephritis. Am J Obstet Gynecol 1991; 164: 587-590.

14. Mazor-Dray E, Levy A, Schlaeffer F, et al. Maternal urinary tract infection: is it independently associated with adverse pregnancy outcome? J Matern Fetal Neonatal Med 2009; 22: 124-128.

15. Renard J, Ballarini S, Mascarenhas T, et al. Recurrent lower urinary tract infections have a detrimental effect on patient quality of life: a prospec tive, observational study. Infect Dis Ther 2014; 4: 125-135.

16. Hooton TM. Clinical practice. Uncomplicated urinary tract infection N Engl J Med 2012; 366: 1028-1037.

17. Beerepoot MA, Geerlings SE, van Haarst EP, et al. Nonantibiotic prophylaxis for recurrent urinary tract infections: a systematic review and meta-analysis of randomized controlled trials. J Urol 2013; 190: 1981-1989.

18. Naber KG, Cho YH, Matsumoto T, et al. Immunoactive prophylaxis of recurrent urinary tract infections: a meta-analysis. Int J Antimicrob Agents 2009; 33: 111-119.

19. Brodie A, El-Taji O, Jour I, et al. A retrospective study of immunotherapy treatment with Uro-Vaxom (OM-89(R)) for prophylaxis of recurrent urinary tract infections. Curr Urol 2020; 14: 130-134.

20. Kotarski J, Nowak-Markwitz E, Poreba R, et al. [Local use of estradiol (Vagifem) in urogynecology - the statement of Polish Gynecological Society experts]. Ginekol Pol 2008; 79: 69-77.

21. Mody L, Juthani-Mehta M. Urinary tract infections in older women: a clinical review. JAMA 2014; 311: 844-854.

22. Albert X, Huertas I, Pereiro II, et al. Antibiotics for preventing recurrent urinary tract infection in non-pregnant women. Cochrane Database Syst Rev 2004: CD001209.

23. Nicolle LE. Uncomplicated urinary tract infection in adults including uncomplicated pyelonephritis. Urol Clin North Am 2008; 35: 1-12, v.

24. Tuncalp, Pena-Rosas JP, Lawrie T, et al. WHO recommendations on antenatal care for a positive pregnancy experience-going beyond survival. BJOG 2017; 124: 860-862.

25. Schneeberger C, Geerlings SE, Middleton P, et al. Interventions for preventing recurrent urinary tract infection during pregnancy. Cochrane Database Syst Rev 2015: CD009279.

26. Matok I, Gorodischer R, Koren G, et al. Exposure to folic acid antagonists during the first trimester of pregnancy and the risk of major malformations. Br J Clin Pharmacol 2009; 68: 956-962.

27. Oplinger $M$, Andrews CO. Nitrofurantoin contraindication in patients with a creatinine clearance below $60 \mathrm{ml} / \mathrm{min}$ : looking for the evidence. Ann Pharmacother 2013; 47: 106-111.

28. Hooton TM, Vecchio M, Iroz A, et al. Effect of increased daily water in take in premenopausal women with recurrent urinary tract infections: a randomized clinical trial. JAMA Intern Med 2018; 178: 1509-1515.

29. Barbosa-Cesnik C, Brown MB, Buxton M, et al. Cranberry juice fails to prevent recurrent urinary tract infection: results from a randomized placebo-controlled trial. Clin Infect Dis 2011; 52: 23-30.

30. Schmidt DR, Sobota AE. An examination of the anti-adherence activity of cranberry juice on urinary and nonurinary bacterial isolates. Microbios 1988; 55: 173-181.

31. Stapleton AE, Dziura J, Hooton TM, et al. Recurrent urinary tract infection and urinary Escherichia coli in women ingesting cranberry juice daily: a randomized controlled trial. Mayo Clin Proc 2012; 87: 143-150.
32. Jepson RG, Williams G, Craig JC. Cranberries for preventing urinary tract infections. Cochrane Database Syst Rev 2012; 10: CD001321.

33. Schwenger EM, Tejani AM, Loewen PS. Probiotics for preventing urinary tract infections in adults and children. Cochrane Database Syst Rev 2015: CD008772.

34. Stapleton AE, Au-Yeung M, Hooton TM, et al. Randomized, placebo-controlled phase 2 trial of a Lactobacillus crispatus probiotic given intravaginally for prevention of recurrent urinary tract infection. Clin Infect Dis 2011; 52: 1212-1217. 